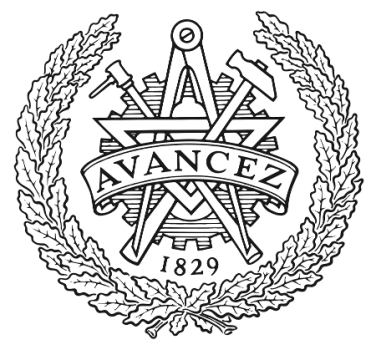

CHALMERS

UNIVERSITY OF TECHNOLOGY

\title{
Systems-level approaches for understanding and engineering of the oleaginous cell factory Yarrowia lipolytica
}

Downloaded from: https://research.chalmers.se, 2023-04-26 10:26 UTC

Citation for the original published paper (version of record):

Poorinmohammad, N., Kerkhoven, E. (2021). Systems-level approaches for understanding and engineering of the oleaginous cell factory

Yarrowia lipolytica. Biotechnology and Bioengineering, 118(10): 3640-3654.

http://dx.doi.org/10.1002/bit.27859

N.B. When citing this work, cite the original published paper. 


\title{
Systems-level approaches for understanding and engineering of the oleaginous cell factory Yarrowia lipolytica
}

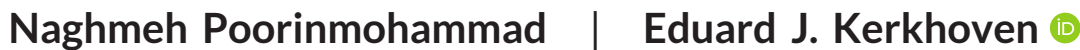

Department of Biology and Biological Engineering, Chalmers University of

Technology, Gothenburg, Sweden

\section{Correspondence}

Eduard J Kerkhoven, Department of Biology and Biological Engineering, Chalmers University of Technology, Kemivägen 10, 41296, Gothenburg, Sweden.

Email: eduardk@chalmers.se

Funding information

Novo Nordisk Fonden, Grant/Award Number: NNF10CC1016517; Research Council for Environment, Agricultural Sciences and Spatial Planning (Formas),

Grant/Award Number: 2018-00597

\begin{abstract}
Concerns about climate change and the search for renewable energy sources together with the goal of attaining sustainable product manufacturing have boosted the use of microbial platforms to produce fuels and high-value chemicals. In this regard, Yarrowia lipolytica has been known as a promising yeast with potentials in diverse array of biotechnological applications such as being a host for different oleochemicals, organic acid, and recombinant protein production. Having a rapidly increasing number of molecular and genetic tools available, $Y$. lipolytica has been well studied amongst oleaginous yeasts and metabolic engineering has been used to explore its potentials. More recently, with the advancement in systems biotechnology and the implementation of mathematical modeling and high throughput omics data-driven approaches, in-depth understanding of cellular mechanisms of cell factories have been made possible resulting in enhanced rational strain design. In case of $Y$. lipolytica, these systems-level studies and the related cutting-edge technologies have recently been initiated which is expected to result in enabling the biotechnology sector to rationally engineer $Y$. lipolytica-based cell factories with favorable production metrics. In this regard, here, we highlight the current status of systems metabolic engineering research and assess the potential of this yeast for future cell factory design development.
\end{abstract}

\section{KEYWORDS}

cell factory, lipid accumulation, metabolic engineering, systems biology

\section{1 | INTRODUCTION}

Limited fossil fuel resources as well as the growing environmental concerns associated with their extraction necessitate a substitute for the traditional chemical synthesis of valuable chemicals. As a renewable and sustainable alternative, microbial biotechnology has been proven efficient to produce high value chemicals (Timmis et al., 2017). Accordingly, an array of microorganisms, from bacteria to yeasts, fungi, and microalgae can be utilized as cell factories to produce a wide range of bioproducts. Among these, Yarrowia lipolytica is increasingly gaining popularity as a biotechnologically relevant cell factory to the extent that it is already regarded to as the most promising nonconventional yeast in bioproduction industry (Markham \& Alper, 2018). Most notably, Y. lipolytica is a well-known oleaginous yeast which can be used to produce lipids and fatty acid-derived bio-based compounds (Blazeck et al., 2014).

Generally, the emerging interest in Y. lipolytica among both industry and academia is further rooted in other advantages such as having GRAS (generally recognized as safe) status (Groenewald et al., 2014), availability of various tools for genetic manipulation (Madzak, 2018), tolerance to different industrial conditions such as different $\mathrm{pH}$ values, higher salt

This is an open access article under the terms of the Creative Commons Attribution License, which permits use, distribution and reproduction in any medium, provided the original work is properly cited.

(c) 2021 The Authors. Biotechnology and Bioengineering Published by Wiley Periodicals LLC 
concentration or the presence of organic acids, high capacity to channe nutrients to commodity chemicals via the efficient flux through the tricarboxylic acid (TCA) cycle (Bilal et al., 2020), and also high potentials in biosynthesis and secretion of proteins (Soudier et al., 2019). Besides being a desirable host for bioproduction, $Y$. lipolytica has also been a model organism for research on the biology and physiology of dimorphism, hydrophobic substrate utilization, protein secretion, and more importantly, lipid metabolism (Beckerich et al., 1998; Beopoulos et al., 2009; Domínguez et al., 2000; Fickers et al., 2005).

Given the broad use of $Y$. lipolytica as an important microorganism with biological relevance and biotechnological applications, significant efforts have been made to expand the knowledge of this yeast. More recently, with the advancements in omics techniques, a quantitative systems-level insight into cellular organizations has made possible to make valuable contributions to bioprocessing (Campbell et al., 2017). Apparently, being able to analyze the entire genome as well as globally quantifying all cellular components and their interactions, does not only expand our knowledge of the biology of the system under study, but also greatly improves the quality of cell factory design. In general, there are multiple biotechnologically important metabolites produced by $Y$. lipolytica and the application of systems metabolic engineering is in its infancy for this yeast. Therefore, it is beneficial to provide an assessment of the state-of-the-art, to evaluate which approaches have let to promising results and where future research should be directed to. Herein, recent advances in the implementation of systems metabolic engineering in optimizing Y. lipolytica as a cell factory is being reviewed.

\section{2 | Y. LIPOLYTICA AS A CELL FACTORY}

The industrial application of $Y$. lipolytica has over half a century of history that began with the discovery of its high lipolytic and proteolytic activity. These features enable the yeast to grow on a diverse range of lipid or protein-rich materials, where many of them can be considered as cheap substrates. The high capacity of lipase secretion was leveraged on by British Petroleum from 1950s through the 1970s, to pioneer the production of single-cell protein (SCP) from $n$-alkanes as substrate (Groenewald et al., 2014). SCP production based on Y. lipolytica is still available as animal feed additive but on a smaller scale (Ritala et al., 2017).

Starting from 2000s and following the development of molecular and genetic tools for engineering the yeast, research studies, as well as commercial productions, have been oriented towards leveraging the abilities of the yeast to produce more than 100 different recombinant proteins, polyalcohols, aromas, emulsifiers, and various organic acids (primarily citric acid) from industrial waste or by-products (LedesmaAmaro \& Nicaud, 2016; Markham \& Alper, 2018; Miller \& Alper, 2019). In addition, the oleaginous phenotype of $Y$. lipolytica has been of interest as it renders the yeast a promising oleaginous cell factory that can produce oils and fatty acid-derived compounds. Two products, New Harvest ${ }^{\mathrm{TM}}$ EPA oil and Verlasso ${ }^{\circledR}$ salmon are commercial examples of this feature and contain omega- 3 fatty acids for which there have been many clinical studies showing a wide range of health benefits (Xie et al., 2015). Generally, oils and fatty acid-derived compounds from $Y$. lipolytica can be used as, for example, biodiesel as well as ingredients for food and cosmetic industries (Miller \& Alper, 2019).

Since lipid biosynthesis is one of the most important features of $Y$. lipolytica as a cell factory, a short overview of general lipid metabolism will be given (also depicted in Figure 1) while the details can be found elsewhere (Fakas, 2017; Lazar et al., 2018). Briefly, triacylglycerol (TAG) lipid species function as storage carbohydrates and can be biosynthesized either de novo or ex novo. The de novo lipid biosynthetic pathway is enhanced by metabolic imbalance upon an essential nutrient limitation in the growth media. In particular, under nitrogen starvation, the carbon flux is redirected towards TAG biosynthesis, by a mechanism where the TCA cycle enzyme isocitrate dehydrogenase is inhibited due to low levels of the allosteric activator adenosine monophosphate (AMP) that is rather broken down to yield ammonium. The ex novo pathway is based on the ability of $Y$. lipolytica to grow on a wide range of hydrophobic carbon sources (Beopoulos et al., 2009). Whether produced de novo or ex novo, lipids can accumulate in a dynamic storage compartment called the lipid body which mainly accommodates neutral lipids such as TAGs, while lipid precursors such as malonyl-CoA can likewise be diverted to alternative products. Metabolism of lipid biosynthesis and accumulation is well studied in Y. lipolytica, comprehensive understanding of which is vital for opting any further systems metabolic engineering approach to improve the production. It is important to note that while wild-type $Y$. lipolytica strains are typically inefficient in de novo lipid biosynthesis from sugars in an industrial scale (Blazeck et al., 2014), various strains have been genetically engineered to reach high lipid contents, where systems-level approaches are found promising in enhancement of production metrics and approaching the maximum theoretical yield.

\section{3 | WET-LAB TOOLS TO FACILITATE SYSTEMS ANALYSIS}

The availability of well-established genome modification techniques is an essential factor for optimizing microbial cell factories. In addition to wide availability of basic genetic engineering tools (i.e., host strains and markers, vectors, promoters, terminators, and replication elements) and conventional genetic modification methods, there is a continuing effort in developing modern gene/genome editing systems for Y. lipolytica that can greatly speed up the application of this yeast in systems biotechnology (comprehensively reviewed in Ganesan et al., 2019). Briefly, from 2016, several efficient CRISPR-Cas9 systems for single and multigene editing as well repression or activation of genes in $Y$. lipolytica are being developed (Gao et al., 2016; C. Schwartz et al., 2017, 2018; C. M. Schwartz et al., 2016; Z. Yang et al., 2020). The gene integration efficiency of up to $55 \%$ has been achieved using a homology-independent targeted genome integration tool mediated by CRISPR/Cas9 which does not require the construction of homologous templates (Cui et al., 2021). More recently, a dual-purpose CRISPR-Cpf1 system has been suggested that is capable of simultaneous gene disruption and 


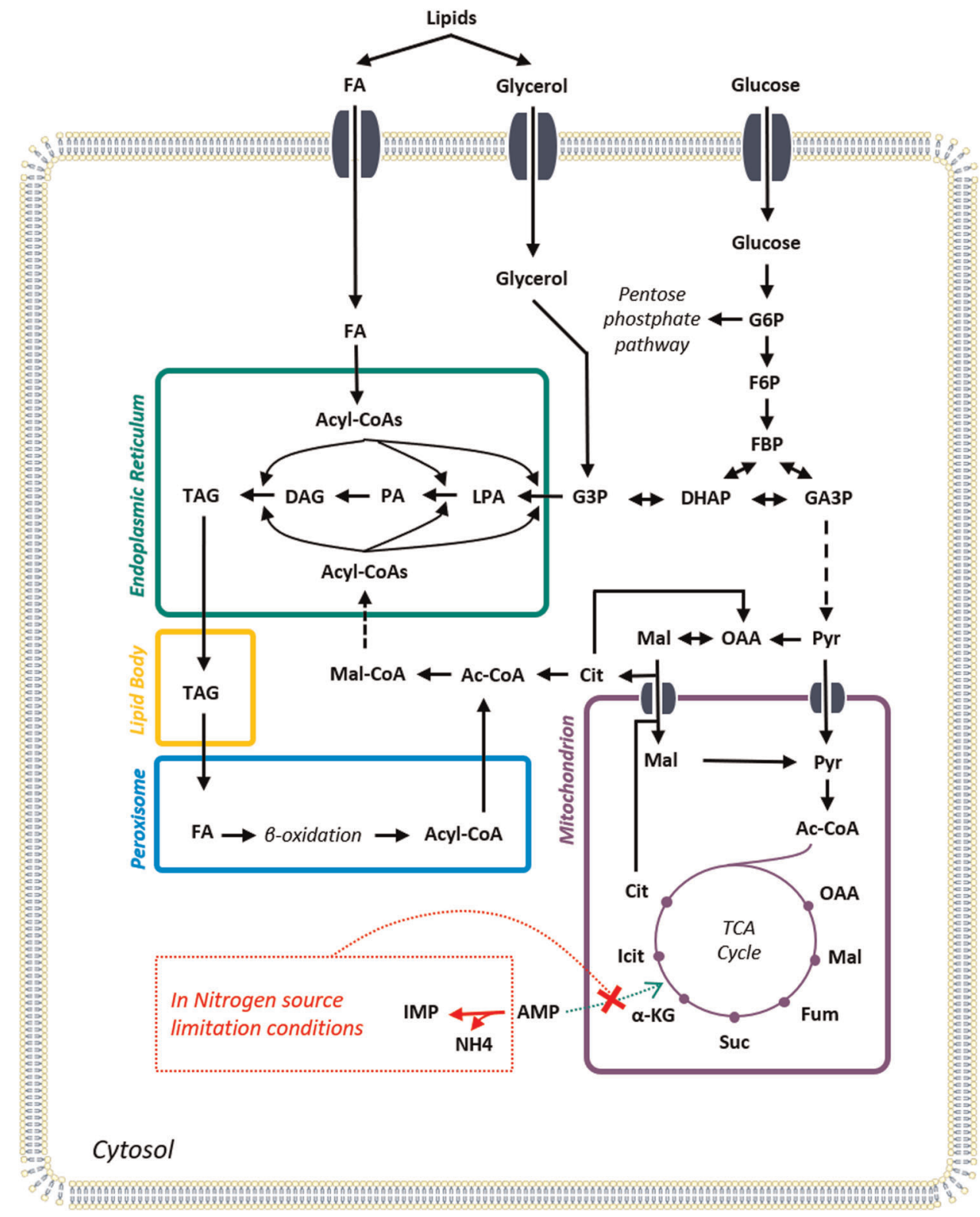

FIGURE 1 Overview of lipid metabolism in Yarrowia lipolytica solid arrows: chemical conversions and transport reactions, dashed arrows: multiple chemical conversion steps, dotted lines and arrows: representing N-limitation consequences. AMP, adenosine monophosphate; Cit, citrate; DAG, diacylglycerol; DHAP, dihydroxyacetone phosphate; F6P, fructose 6-phosphate; FA, fatty acid; FBP, fructose 1,6-bisphosphate; Fum, fumarate; G3P, glycerol 3-phosphate; G6P, glucose 6-phosphate; GA3P, glyceraldehyde 3-phosphate; Icit, isocitrate; IMP, inosine monophosphate; LPA, lysophosphatidic acid; Mal, malate; Mal-CoA, malonyl coenzyme A; NH4, ammonium; OAA, oxaloacetate; PA, phosphatidicacid; Pyr, pyruvate; Suc, succinate; TAG, triacyglycerol [Color figure can be viewed at wileyonlinelibrary.com]

gene regulation in Y. lipolytica (Ramesh et al., 2020). Furthermore, a suite of genetic tools (EasyCloneYALI) has been generated that facilitates transformation protocols by providing a series of predesigned plasmids and oligos (Holkenbrink et al., 2018). There is still a significant opportunity to further improve CRISPR-based systems in Y. lipolytica in terms of gene integration efficiency (similar to gene disruption efficiency of about $90 \%$ ), multigene editing efficiency or the need for a high-throughput screening technology after genome editing. Although the application of the CRISPR-Cas9 usage and its innovative strategies in the model yeast, Saccharomyces cerevisiae, shed light and laid the foundation of CRISPR-Cas9 implementation in other nonconventional yeasts technology, its optimizations need species-specific studies. However, there are several general factors that have been proven in this reference yeast to positively affect the efficiency of CRISPR-Cas9 genome modifications amongst which using weak to a medium-strength promoter for expressing CAS9, a priority of using natural RNAP III promoters, careful choice of the gRNA binding site and taking advantage of in silico tools for sgRNAs 
design to decrease off-target effect can be named (Cai et al., 2019; Raschmanová et al., 2018). When optimized, these systems provide a suitable platform to be coupled with the systems-level tools to further promote the systems metabolic engineering process.

Meanwhile, and in parallel with studies to optimize CRISPR-Cas9 systems for $Y$. lipolytica, other genome modification strategies are being developed mainly due to the little information on CRISPR-Cas9 related molecular tools in this yeast. For instance, a Cre/lox-site-specific recombination system using only a single selection marker has been developed for targeted, repeated, and marker-less gene integration in $Y$. lipolytica with above $90 \%$ efficiency. Unlike similar previous methods based on Cre/lox-site-specific recombination, their genetic tool was also proved effective for various genes, loci, and strains which suggests a promising application of $\mathrm{Cre} / \mathrm{lox}$ system and strengthens its capabilities for genome modification in Y. lipolytica (Zhou et al., 2021). Moreover, as a study to overcome the challenge with building complex pathways and multiple gene integration in Y. lipolytica, Z. Guo et al. (2020) have designed a completely synthetic $Y$. lipolytica-specific artificial chromosome (yIAC) and successfully used it to introduce a complete cellobiose and xylose co-utilization pathway introduced to the yeast in a single step within less than one week of wet lab experimentation. The method thus is much faster and efficient in comparison to conventional multistep procedures and can be applied for multigene integration in $Y$. lipolytica. Based on existing efforts to create orthogonal systems where the synthetic biology parts of one yeast have been applied to another (Patra et al., 2021), it is expected that in near future, the synthetic biology tools and techniques will be applied interchangeably among different species.

In all, recent advances have provided Y. lipolytica with a plethora of genetic engineering and genome editing techniques (Holkenbrink et al., 2018; Madzak, 2018), widening the perspectives for systems metabolic engineering of $Y$. lipolytica as a potential biotechnological workhorse.

\section{4 | SYSTEMS METABOLIC ENGINEERING OF Y. LIPOLYTICA}

The functional genomics revolution and increasing ease of omics data generation and computational analysis aids in understanding the mechanisms of complex biological systems and the prediction of their dynamic properties via systems biology. This paradigm of studying a cell as a whole is being efficiently implemented to provide a holistic view of the functioning of microbial cell factories with the goal of rational re-designing or creating new cellular functions in these organisms.

Under the moniker of metabolic engineering, modifications of biochemical pathways have been used for decades to achieve desired phenotypes such as overproduction of a metabolite in cell factories, either using random mutagenesis or other conventional molecular genetic engineering tools. However, only after the development of the methods and tools in the discipline of systems biology has it become practical to approach the metabolic engineering challenges from a systems perspective. The development of an optimal strain via systems metabolic engineering can be performed through two major approaches: omics data-driven (top-down) and biomolecular modeldriven (bottom-up). While the bottom-up approach starts with the reconstruction of an accurate mathematical model of a specific biomolecular subsystem (i.e., metabolic, gene regulatory and signaling networks, or combinations thereof) using existing biological knowledge; the top-down approach begins with extracting information from relevant omics data sets (e.g., metabolomics, proteomics, transcriptomics, and fluxomics). Figure 2 illustrates these strategies as well as the classic metabolic engineering.

During the past decade, there have been multiple studies on biomolecular model analysis and omics data generation that aimed to improve the understanding and production of many valuable metabolites in Y. lipolytica. Genome-scale metabolic models (GEMs) are the most commonly studied biomolecular networks, the popularity of which arises not only from their innate nature which directly considers the analysis of the metabolite production phenotype, but also from the availability of powerful constraint-based modeling methods. As GEMs can provide a global view of cellular metabolism, they are influential tools in systems metabolic engineering. Currently, there have been six GEMs published for Y. lipolytica by different research groups, where each model includes improvements over previously published models. All six GEMs have been used for evaluating metabolic engineering strategies with a major focus on over-producing lipids, for example, triacylglycerols (TAGs) and to a lesser extent for the increased dicarboxylic acids production (Kavšček et al., 2015; Kerkhoven et al., 2016; Loira et al., 2012; Mishra et al., 2018; Pan \& Hua, 2012; Wei et al., 2017).

Since obtaining high-quality omics data has become progressively easier, interest in applying data-driven approaches for acquiring systems-level knowledge of $Y$. lipolytica have increased (Ledesma-Amaro \& Nicaud, 2016). While the availability of complete genome sequences reveal its metabolic pathways and functional potential, various transcriptomics and a few proteomics analyses have provided useful information about the dynamics of biological processes, such as the transition from the growth phase to the lipid accumulation phase as well as its regulatory mechanisms (Morin et al., 2011). There have also been a few metabolomics and lipidomics analyses in Y. lipolytica, which further provide information on the metabolic pathways under study by incorporating the changes in metabolite concentrations (Zhao et al., 2015).

As individual omics analyses (e.g., transcriptomics or metabolomics) are not always able to fully elucidate the behaviors of a cellular system, combining different omics data (e.g., transcriptomics and metabolomics) may result in a more precise picture of the biological system being studied. Moreover, integration of various types of omics data following a bottom-up approach can boost the predictive capability of the model (Gu et al., 2019). Such combinatory approaches have been applied in a few studies in metabolic engineering of $Y$. lipolytica.

Generally, examples of systems-level understanding as well as metabolic engineering efforts in Y. lipolytica powered by systems biology knowledge and tools will be discussed below in relative 


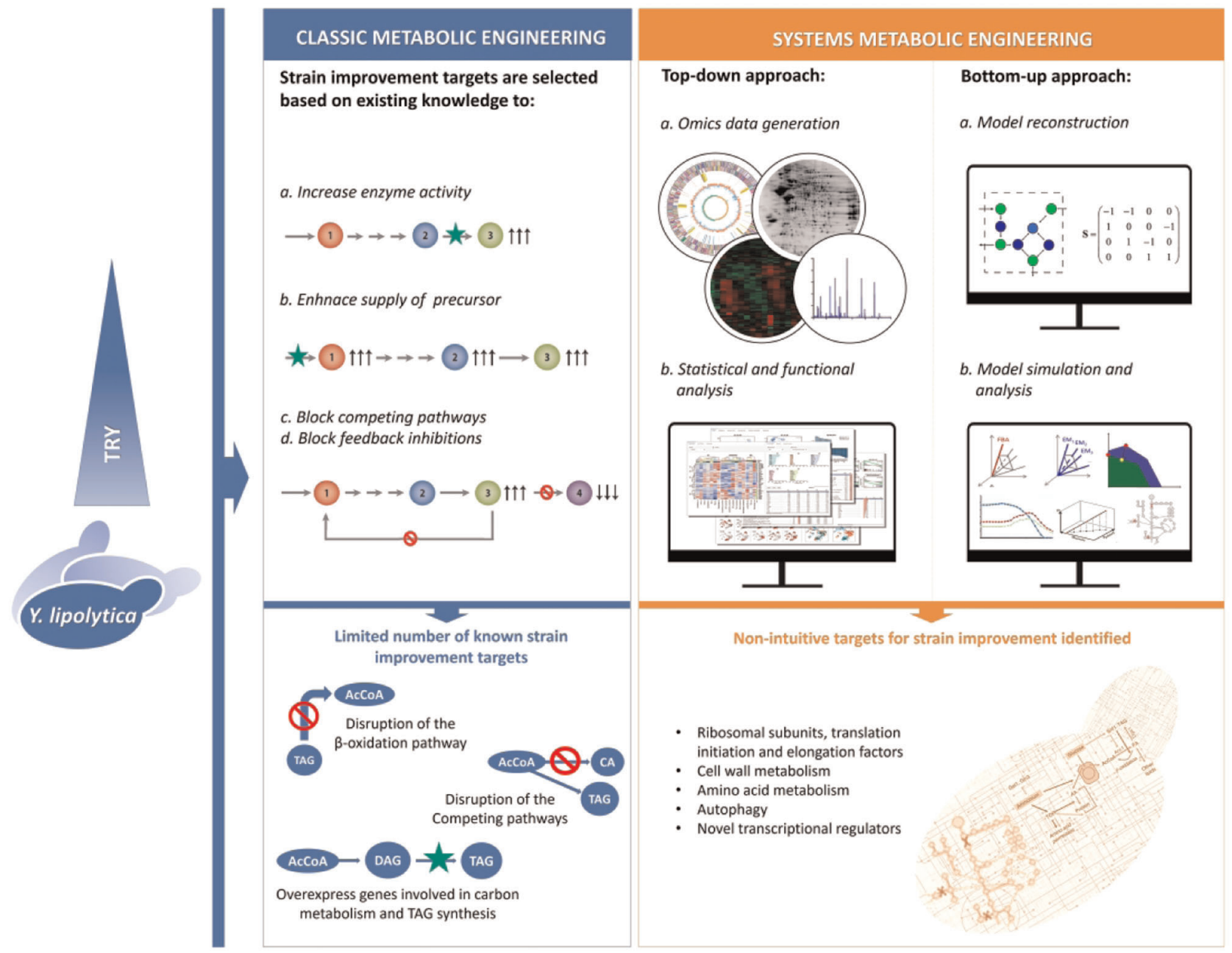

FIGURE 2 Schematic procedure of classic versus systems metabolic engineering and the major identified engineering targets for lipogenesis in Yarrowia lipolytica by each strategy [Color figure can be viewed at wileyonlinelibrary.com]

sections. Furthermore, Table 1 summarizes the systems-level optimizations of lipid-derived as well as nonlipid products in Y. lipolytica.

\subsection{Lipids and fatty acid-derived products}

Various studies have been performed to gain a systems-level understanding of lipid metabolism with focus on three main questions: the main source of NADPH that lipid accumulation demands; gene regulation that coincides with lipid accumulation; and transcription factor networks that are underlying the expressional changes during lipid accumulation as will be detailed below. In many cases, such studies have not only expanded our knowledge of various aspects of lipid metabolism, but also yielded proposed targets for genetic engineering for improved lipid production.

Lipid biosynthesis has a high NADPH demand that could theoretically be provided through various metabolic pathways. Based on a comparison of flux distribution obtained by ${ }^{13} \mathrm{C}$ metabolic flux analysis in two strains of $Y$. lipolytica, one of which was engineered to produce lipids at roughly twice the yield of the wild strain,
Wasylenko et al. (2015) concluded that the oxidative pentose phosphate pathway (PPP) is the almost exclusive source of lipogenic NADPH when Y. lipolytica is grown on glucose. However, when cultured on acetate, another flux distribution analysis by N. Liu et al. (2016) showed the importance of gluconeogenesis on lipogenic NADPH synthesis.

To reveal if lipid accumulation is transcriptionally regulated, the first transcriptomics analysis of $Y$. lipolytica during its metabolic shift from growth to lipid accumulation indeed showed significant difference between the expression profile of actively dividing and lipid accumulating cells (Morin et al., 2011). However, the genes directly governing fatty acid synthesis were not transcriptionally regulated, but rather, the authors hypothesized that lipid accumulation is a passive consequence of the rerouting of carbon fluxes. Upon nitrogen deprivation a cascade of transcriptional events is triggered that result in an increased supply of precursor and cofactors necessary for the synthesis of lipids. Subsequent transcriptomics as well as multiomics studies confirmed this hypothesis and elucidated the regulatory role of protein and amino acid metabolism in lipogenesis rather than the transcriptional regulation of lipid metabolism itself. 


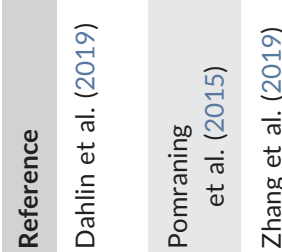

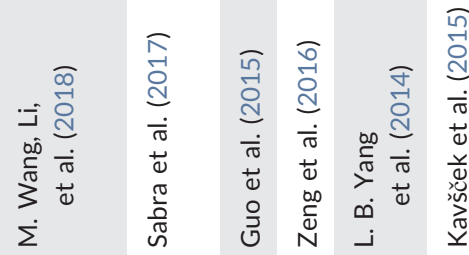

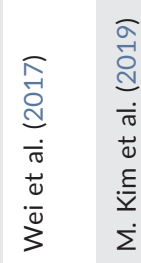
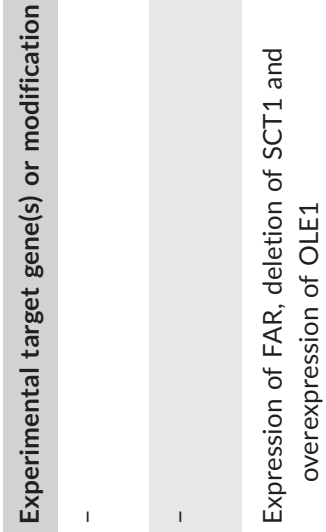

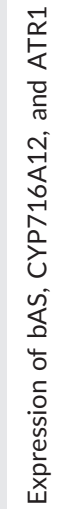
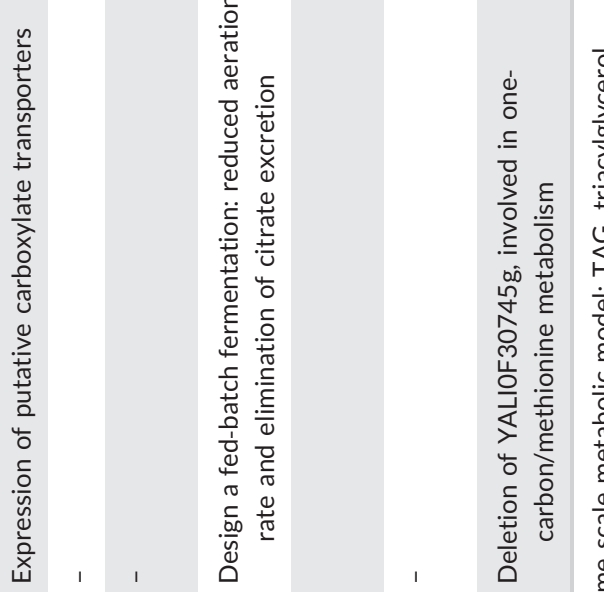

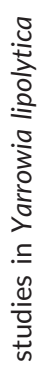

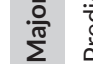

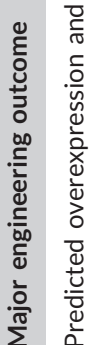

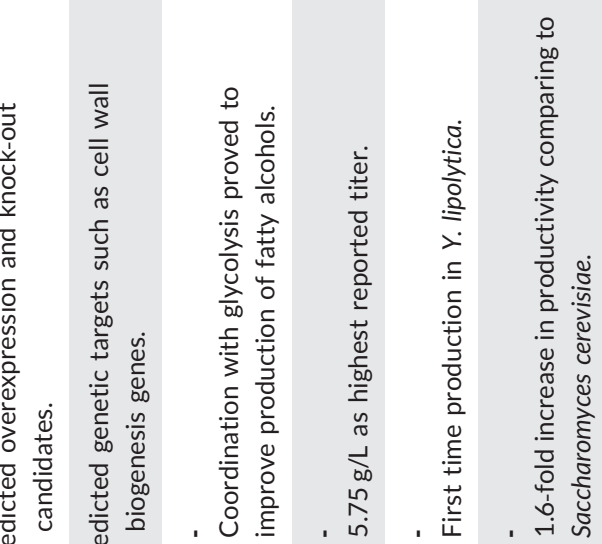

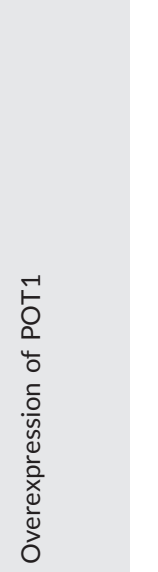

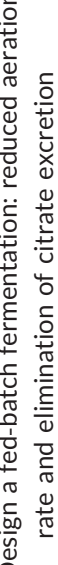

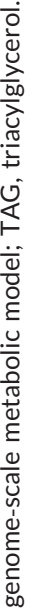

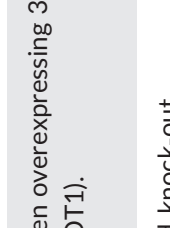

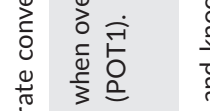

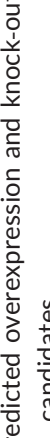

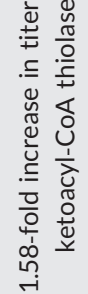

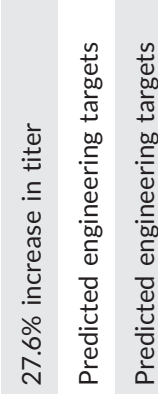

.

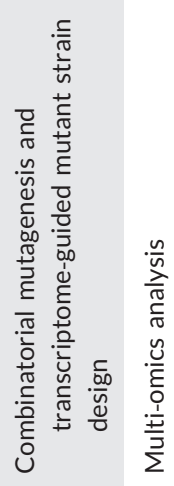

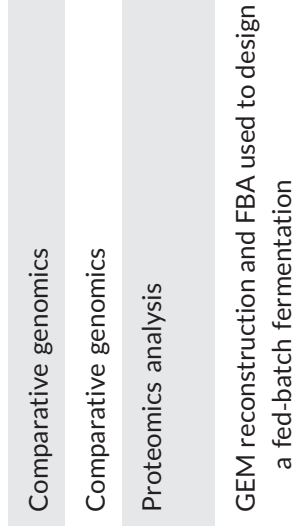

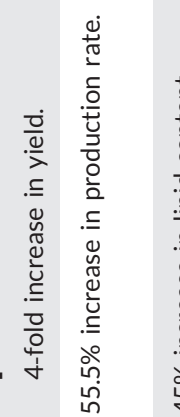

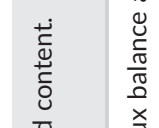

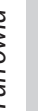

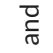

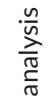

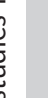

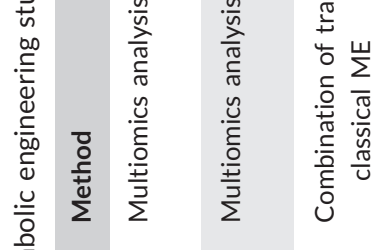

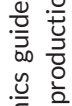

용

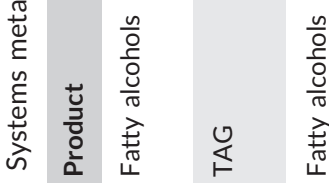

$-\frac{0}{0}$
$\frac{0}{0}$
$\frac{0}{0}$
$\frac{0}{0}$
$\frac{0}{0}$

童
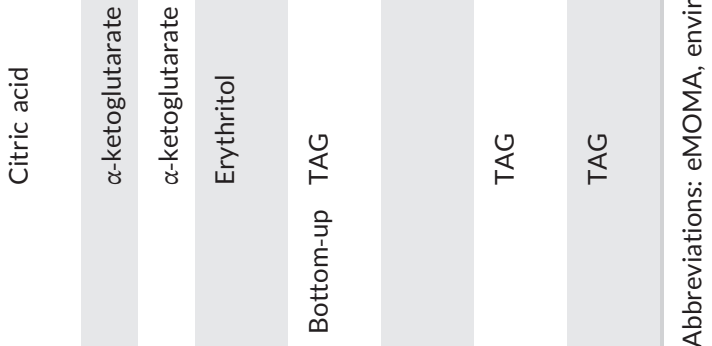
By integrating proteome, phosphoproteome and metabolome data, Pomraning et al. (2016) suggested posttranslational modification of key lipogenesis enzymes as the governing regulatory mechanism. Specifically, ATP-citrate lyase and acetyl-CoA carboxylase (ACC) were not differentially expressed but their level of phosphorylation increased when cells were grown in media with low nitrogen availability, suggesting that this contributes to increased lipid accumulation, which would be orthologous to the phosphorylation sites of $S$. cerevisiae Acc1 that have been leveraged to enhance lipogenesis in that species (Shi et al., 2014). In Y. lipolytica, later studies showed that genes related to branched-chain amino acid metabolism were downregulated upon nitrogen limitation, while alternative nitrogen supply pathways such as protein turnover and autophagy genes were upregulated among which an important factor that affects lipid synthesis was reported to be the metabolism of leucine (Kerkhoven et al., 2016, 2017). These findings were similar the results from a multiomics study of another oleaginous yeast, Rhodosporidium toruloides (Zhu et al., 2012). Here, the transcriptomic and proteomic data suggest that lipid accumulation under nitrogen-limited conditions correlates with the macromolecule metabolism and autophagy which may denote to similarities in the mechanisms of microbial oleaginicity. In an independent research where links between disruption of the MHY1 gene, involved in dimorphic transition in $Y$. lipolytica, and lipid accumulation have been studied, transcriptome data from the MHY1 mutant strain showed decreased amino acid biosynthesis while lipid accumulation increased by $13 \%$ based on cell dry weight (G. Wang et al., 2018).

To elucidate if regulatory networks are underlying lipid accumulation, Trébulle et al. (2017) inferred a genome-wide coregulatory network using CoRegNet on the basis of the gene expression data during lipid accumulation under nitrogen limitation. According to their reconstructed regulatory network, network modules representing different transcriptional programs, from growth to lipid accumulation phases, along with the most influential new transcription factors (TFs) and co-regulators have been identified. Furthermore, the role of the top candidates on lipid accumulation was validated experimentally using a high throughput overexpression system (Leplat et al., 2018). Based on the identified regulators and target genes, their findings again support the hypothesis that lipid accumulation is a consequence of change in carbon fluxes rather than an enhanced lipid metabolism (Trébulle et al., 2017). Accordingly, the tuning of key regulators can effectively facilitate lipid accumulation by enhancing the carbon flux. Different regulators have been identified in this regard. For instance, in separate studies deletion of glucose repression regulators SNF1 and MIG1, have resulted in 2.6- and 1.5 -fold increase in lipid content of $Y$. lipolytica, respectively (Seip et al., 2013; Z.-P. Wang et al., 2013). Through reducing the flux to TCA cycle by imbalancing the TCA cycle intermediates, deletion of UGA2 have also resulted in 1.5-fold enhanced lipid content (L. Liu et al., 2015). As another example of tuning carbon flux via regulators is the mutation into Mga2p, a regulator of desaturase gene expression, which resulted in enhances lipid titer due to imbalance caused between glycolysis and the TCA cycle (L. Liu et al., 2015). It is thus noteworthy that effective systems biology approaches such as multiomics studies (e.g., integration of transcriptome and metabolome data) integrated with in silico metabolic model simulations could be used to analyze regulatory dynamics (Hubbard et al., 2018), and from this transpired that lipid accumulation is not merely an effect of isocitrate dehydrogenase inhibition during nitrogen starvation.

Beyond employing systems-level approaches to gain understanding on lipid metabolism, it has been used to find systems-level solutions that favor the oleaginous phenotype in terms of improving its yield, productivity, and titer as well as broadening the substrate range. Nonetheless, most studies that have improved lipid production can be classified as classical metabolic engineering, via overexpression of genes that are directly involved in lipid biosynthesis, deletion of those involved in lipid degradation, expressing genes required for the consumption of different carbon sources, balancing cofactors, as well as a combination of these strategies which are comprehensively discussed elsewhere (Abdel-Mawgoud et al., 2018; Das et al., 2020; Lazar et al., 2018). While rationally designed classical metabolic engineering has been able to push lipid accumulation to laudable levels, systems-level approaches would be able to also reach the uncharted territories of the lipogenesis metabolic landscape that would harbor a strain that is robust, versatile, and pushes lipid accumulation to its limits. Systems metabolic engineering efforts in $Y$. lipolytica have therefore aimed to approach the theoretical maximal yield via understanding the less intuitive limiting factors and based on conclusions formulated in omics and model-based studies.

Accordingly, using a bottom-up approach through the analysis of a manually reconstructed GEM for Y. lipolytica (iMK735), Kavšček et al. (2015) suggested a positive effect of oxygen limitation on lipid production in the productive phase giving the hypothesis that reduced aeration rate, which is also favored in industry, might induce lipid accumulation. Moreover, to eliminate citrate excretion, a reduced glycolytic flux was calculated while maintaining lipid synthesis rate. Combination of these findings were used to design a fermentation strategy which resulted in $80 \%$ increase in the lipid content of biomass and more than fourfold yield improvement (Kavšček et al., 2015). In a later model-based approach, various gene knockouts and overexpression targets for improved TAG biosynthesis were predicted by analyzing a comprehensive GEM reconstructed by Wei et al. (2017) Although not experimentally verified, gene modification strategies mostly being involved in glycolytic and amino-acid metabolic pathways were predicted to yield over $55 \%$ increase on TAG production. In a most recent model-based study, a modified version of iMK735 was implemented to analyze flux distributions of $Y$. lipolytica in nitrogen-limited conditions as well as identify metabolic engineering strategies for improving lipid production. Since the cellular objective of growing cells during nitrogen limitation is not obvious, an environmental version of minimization of metabolic adjustment (eMOMA) was used instead of the conventional flux balance analysis (FBA) that requires an assumption of the cellular objective, for example, maximized biomass production. This modeling approach predicted multiple novel and nonintuitive targets for metabolic engineering while experimental verification of one of the knockout candidates involved in one-carbon/methionine metabolism 
(annotated as methylenetetrahydrofolate dehydrogenase) showed 1.45-fold increase in lipid accumulation (M. Kim et al., 2019).

As the current GEM-based analyses rely on constraint-based analysis of the metabolic network stoichiometry, these models are limited in providing insight into regulatory relationships, rate-limiting steps, or the effects of altering enzyme expression. Noteworthy is that the current GEM-based analyses did not include any regulatory information. To address this limitation, kinetic models that quantitatively describe the metabolic and regulatory processes through kinetic formalisms (e.g., mass action, Michaelis-Menten; Foster et al., 2021). Few examples of such models exist among nonconventional yeast. In this regard, Robles-Rodriguez et al. have built and statistically validated three dynamic metabolic models of Y. Iipolytica to describe its lipid accumulation and citric acid production while growing on glucose (Robles-Rodriguez et al., 2017). Although, no experimental validation has been sought, their results showed acceptable fit of parameters so that they can potentially be implemented to optimize lipid accumulation.

Despite the discussed progress, the power of genome-scale modeling is still limited for a biotechnologically favorable overproduction thus, the result of genetic interventions using this approach needs critical evaluation. In case of lipogenesis, this is also since the lipid metabolism has proven to be quite complex in case of $Y$. lipolytica and it indicates the need of incorporating more data into genome-scale models as discussed before. Moreover, multiple approaches can be used to overcome this issue amongst which bioprocess engineering $(\mathrm{H}$. Liu et al., 2020), use of mixed substrates to optimize metabolism (N. Liu et al., 2020), and cellular engineering (Soong et al., 2019) can be named, discussion on which is out of the scope of this review. However, implementing such approaches along with existing metabolic engineering methods can potentially enhance cell performance.

Generally, top-down omics-based approaches have not only augmented the biological knowledge of lipid biosynthesis, but also in some cases non-experimentally verified suggestions for production improvement have been concluded from these analyses. Of the nonintuitive examples of these suggestions is the role of cell wall biosynthesis genes in lipid production, shown by Pomraning et al. (2015) where cell wall thickening was observed upon the exhaustion of extracellular carbon source. Omics data such as fluxomics, intracellular metabolomics, and proteomics can greatly help in understanding of the regulation of lipid metabolism, which in turn result in targets for metabolic engineering of $Y$. lipolytica for the production of lipids and fatty acid-derived products. In all, more thorough combination of top-down and bottom-up approaches would therefore be recommended.

\section{2 | Specific oleochemicals}

While classic and systems metabolic engineering efforts continue to improve lipid production in Y. lipolytica by maximizing flux through TAG biosynthesis, production of specific valuable oleochemicals are being studied in this yeast mostly via synthetic pathway construction.

Many commercially interesting oleochemicals, for example, medium-chain lipids, are less common in natural resources and are therefore ideal candidates for systems and synthetic biologybased production due to their high value (Blazeck et al., 2014). These oleochemicals can be used as nutraceuticals or pharmaceuticals, such as polyunsaturated fatty acids (PUFAs) or fatty alcohols (Ledesma-Amaro \& Nicaud, 2016). Substantial progress has been made towards heterologous production of such oleochemicals in Y. lipolytica, but only recently have conclusions from systems-level analysis been implemented to improve the production of nonnative oleochemicals. Most of these efforts are through using the top-down strategy. For instance, in a study by Dahlin et al. (2019), a lipid degradation-deficient strain of Y. Iipolytica was used as host organism to express copies of codonoptimized fatty acyl-CoA reductase from Marinobacter algicola. The final fatty alcohol producer strain was evaluated by transcriptomics, metabolomics, and fluxomics analysis. According to the results, unlike $S$. cerevisiae, no elicited stress response was detected in $Y$. lipolytica indicating the non-toxicity of fatty alcohols in this strain. Moreover, potential limiting factors upstream of acetyl-CoA, such as glucose uptake, glucose phosphorylation, conversion to fructose-6-phosphate/fructose-1,6-bisphosphate as well as pyruvate and citrate were indirectly predicted based on metabolomic analysis (Dahlin et al., 2019). Transcriptomics of another fatty alcohol producing Y. lipolytica aided to significantly improve the production upon identifying the upregulation of glycolysis genes in response to higher fatty alcohol production. This result was leveraged to optimize the selection of suitable promoters for fatty acyl-CoA reductase expression, to coordinate the fatty alcohol production with glycolysis (Zhang et al., 2019).

To repurpose the lipophilic properties in Y. lipolytica for betacarotene biosynthesis, Wang et al. designed a strategy of global transcription machinery engineering (gTME) to explore the changes of gene expression levels that benefits carotene production ( $\mathrm{M}$. Wang, Li, et al., 2018). This strategy had been introduced for other complex cellular genotypes in S. cerevisiae (El-Rotail et al., 2017) and Zymomonas mobilis (Tan et al., 2016). Accordingly, a library of randomly mutated versions of the key transcription factor YI-Spt15 were expressed in a strain containing a heterologous beta-carotene biosynthetic pathway. Strains with mutated YI-Spt15 expression displaying either enhanced or decreased production were analyzed by transcriptomics, and the most dominant differentially expressed genes were experimentally tested and those involved in RNA polymerase, fatty acid metabolism, and specially ketone body metabolism were shown to be beneficial for beta-Carotene production improvement. Although the reported beta-carotene yields were far lower compared to other published yields, their exploited gene targets and pathways can further guide the rational design of Y. lipolytica for tuning lipophilic production in combination with improved fermentation design. 


\subsection{Nonlipid products}

Although a major focus in Y. lipolytica research is on lipogenesis, this yeast is able to produce other native and nonnative industrially important chemicals such as organic acids (OAs) and polyols. The most industrially important nonlipid chemicals are OAs which are direct or indirect intermediates of the TCA cycle. Due to its oleaginous phenotype, Y. lipolytica accommodates a highly active TCA cycle. However, since biosynthesis of OAs is highly linked to cellular energy metabolism, reaching efficient production metrics will require more complex engineering approaches. This renders systems metabolic engineering profitable tools to optimize OA production. Nonetheless, also for this group of products has classical metabolic engineering through rational genetic modifications and optimization of fermentation condition shown to enhance the productivity (reviewed in Finogenova et al., 2005). Meanwhile, efforts to elucidate the underlying systems-level production mechanisms are crucial to optimize cell metabolism favorable for OA production. In terms of citric acid (CA) production, Sabra et al. (2017) have focused on transcriptome and fluxome data in a citrate producing strain of $Y$. lipolytica. While controlling the oxygen supply on mixed substrates proved instrumental to improve the citrate production titer more than three fold, multiomics data analysis have provided pentose phosphate pathway and glyoxylate cycle genes as targets for metabolic engineering of CA (Sabra et al., 2017). In another study, after reporting $Y$. lipolytica as the best CA producer among more than 40 yeast strains, Kamzolova et al. (2017), studied the underlying overproduction mechanism using growth limitation assessment and enzyme activity measurements which helped in fermentation design for enhanced production $(85 \mathrm{~g} / \mathrm{L})$. A more recent screening also showed that the production of $\mathrm{CA}$ strongly strain dependent, therefore, screening for overproducers and studying the underlying molecular mechanisms is of great importance (Carsanba et al., 2019). Noteworthy is that due to the relations between lipid and CA metabolism, knowledge of the regulation surrounding lipid accumulation as well as nitrogen limitation can have considerable relevance for CA production studies.

The production of succinic acid (SA) in Y. lipolytica has also undergone metabolic engineering efforts (Abdel-Mawgoud et al., 2018). Unlike SA-producing bacteria, which are among the most successful producers to date, Y. lipolytica can tolerate low $\mathrm{pH}$ and thrives under acidic conditions. This simplifies downstream processes as the bacterial fermentation broth is rather kept near-neutral $\mathrm{pH}$ and requires an additional acidification step for SA purification which causes byproduct formation (Ahn et al., 2016). Although high titers have been achieved $(160 \mathrm{~g} / \mathrm{L})$ in an engineered strain lacking active succinate dehydrogenase (SDH) to prevent oxidation of succinic acid to fumaric acid, low yield and productivity hinders the implementation of $Y$. lipolytica for industrial production. SDH deficient mutants of $Y$. lipolytica are growth defective in cultures using glucose source (Yuzbashev et al., 2010). To circumvent this problem Yuzbashev et al. have isolated an improved SA producer from combined induced mutagenesis and metabolic evolution, which could more efficiently utilize glucose (Yuzbashev et al., 2016). ${ }^{13} \mathrm{C}$ flux analysis on SDH deficient mutants was performed to investigate the contribution of various metabolic pathways in SA formation (Yuzbashev et al., 2016), however, to fully reveal the molecular mechanism by which adaptive evolutionary approaches have resulted in a beneficial phenotype would require genome sequence analysis of the evolved and unevolved strains. A further problem hindering improvement of SA production is the accumulation of the by-product acetate (Cui et al., 2017). By metabolic pathway analysis, Cui et al. have reported CoA-transferase activity in mitochondria as the major source of acetic acid. Deletion of the responsible gene, as well as further overexpression of the key enzymes of the oxidative TCA resulted in an improved SA titer and no acetic acid accumulation in defined media (Cui et al., 2017). There have been successful systems metabolic engineering approaches for SA production in other microorganisms, such as GEM-based SA production improvement on S. cerevisiae (Agren et al., 2013) as well as other omics-based studies (Otero et al., 2013). Similar approaches could be applied to $Y$. lipolytica to yield industrially feasible SA production levels in this yeast.

$\alpha$-ketoglutarate $(\alpha-K G)$ is another OA for which $Y$. lipolytica seems a promising production host. The main strategy being used to improve its production is still based on fermentation design as well as a few genetic engineering in the direct biosynthetic enzymes (H. Guo et al., 2016). Therefore, while systems-level insight is still desired to investigate key metabolic processes influenced and regulated by the known factors that positively affect $\alpha-K G$ production, there are a few studies based on omics data. In a study to improve $\alpha-K G$ production, Guo et al., have assessed the roles of specific transporters (H. Guo et al., 2015). To this end, they have identified six endogenous putative transporter genes using a comparative genomics approach and by expressing them in an $\alpha$-KG-producing wild-type strain, the production of this OA has improved while reduced pyruvic acid (a byproduct) concentration was observed. Their study showed whole genome analysis for transporters can be helpful in improving the accumulation of specific organic acids. Another comparative genomics method with the aim of investigating the regulatory mechanisms controlling $\alpha-K G$ production, showed the positive role of genes associated with the regulation of $\mathrm{mi}$ tochondrial biogenesis and energy metabolism in $\alpha$-KG production, whereas they have concluded genes related to transformation between keto acids and amino acids as reducers of the accumulation of $\alpha-K G$ (Zeng et al., 2016). As another strategy to accumulate $\alpha-K G$, is to take advantage of its thiamineauxotrophic trait. Y. lipolytica is unable to biosynthesize thiamine which is the co-factor for $\alpha$-ketoglutarate dehydrogenase which converts $\alpha$-KG to succinyl-CoA. Therefore, a culture medium lacking this vitamin will likely favor the accumulation of $\alpha-K G$ 
when carbon source is in excess. However, enhanced accumulation of $\alpha-K G$ by disruption of genes directly affecting TCA cycle will undoubtedly cause cellular growth and survival defects circumventing which urges deeper molecular knowledge. In this regard, similar omics data-based findings of the study by Walker et al. on the effects of thiamine deficiency on cellular metabolism of thiamine auxotroph Y. lipolytica which was discussed in lipid production improvement strategies, can be helpful (Walker et al., 2020).

Beyond native OAs, Y. lipolytica has been used to produce the nonnative itaconic acid (IA). IA is naturally produced by several Aspergillus species and is a replacement for petroleum derived products (Robert \& Friebel, 2016). Due to shortcomings with the native producer in terms of poor growth and shear stress sensitivity, its production is studied in alternative hosts such as $Y$. lipolytica. IA is synthesized by cis-aconitic acid decarboxylase (CAD) (Kanamasa et al., 2008) and IA production in Y. lipolytica has been established by heterologous expression of CAD (Blazeck et al., 2015) resulting in IA yields of $0.06 \mathrm{~g} /(\mathrm{g}$ glucose) at natural low $\mathrm{pH}$. Although these studies can serve as a promising stepping-stone toward enhancing IA production in $Y$. lipolytica, to establish this yeast as an industrially competitive IA producer, further efforts are required to improve production metrics. Even while bottom-up systems-level approaches have been used for IA production in other microorganisms (Harder et al., 2016), there is still no report on systems metabolic engineering in Y. lipolytica.

Besides OAs, Y. lipolytica is a promising producer of sugar polyols, and there is a currently strong interest in bio-based production of such value-added functional sugars for use in food and pharmaceuticals industry as sweetener (e.g. mannitol and erythritol). Recent progress in the use of $Y$. lipolytica to synthesize these functional sugars, especially the genetic engineering approaches, are comprehensively reviewed elsewhere (Bilal et al., 2020). The importance of systems-level studies in this field has been demonstrated in a study by Yang et al. where erythritol production could be enhanced in an osmophilic strain of $Y$. lipolytica by cultivation in an osmotic pressure control fed-batch strategy (L. B. Yang et al., 2014). Proteomics of erythritol production from glycerol in response to osmotic pressure revealed that highly ranked differentially-expressed proteins such as enzymes related to osmotic stress response, the aldo-keto reductases and catalase $\mathrm{T}$ had drastically increased expression levels under hyperosmotic pressure (L. B. Yang et al., 2015). This information could be leveraged on for future reverse engineering of $Y$. lipolytica strains for polyols biosynthesis.

Production of many pharmaceuticals and nutraceuticals employing Y. lipolytica as a host has been studied mostly using classic metabolic engineering (Zhang et al., 2020). Oleanolic acid is pentacyclic triterpenoid with antioxidation activity and its heterologous production have been studied in Y. lipolytica. Based on intracellular metabolome data, a direct precursor of the triterpenoid was found to be accumulating. Guided by the analysis of differential transcriptional levels of genes, it transpired that enhancement of electron transfer by fusion expression of P450 enzyme and the last step of the biosynthetic pathway could be used to improve substrate conversion efficiency by $28 \%$ (Li et al., 2020).

\section{5 | CONCLUSIONS AND PERSPECTIVES}

For decades, Y. lipolytica has been known as a promising biotechnological chassis not only for its high oleaginous flux, but also for the production of a variety of native and nonnative chemicals. However, despite advances in genome editing tools which have facilitated classical metabolic engineering of $Y$. lipolytica, production metrics for most products still needs improvement. Questions remain on how to overcome limitations in growth characteristics, substrate degradation and product formation. In this regard, systems biology allows a more in-depth understanding of the intricate regulation and metabolism which can further help in identifying nonintuitive strain design targets with the aim of pushing yields to the theoretical limits. This can in turn expand the industrial potential of this yeast. Although several $Y$. lipolytica strain engineering studies have begun to involve omics and model-based approaches, there is significant potential in further use of effective systems-level approaches. Moreover, systems-level approaches have been used for studying lipidderived products with some promising results, while non-lipid products have benefited very little. Furthermore, both bottom-up and top-down approaches are used, although not to the same extend in all the products of $Y$. lipolytica. A number of specific next steps can be identified to push this field further.

\section{1 | Combination of top-down and bottom-up approaches}

As classical metabolic engineering results typically show, the overexpression of a gene target and in vivo production improvement are rarely linearly correlated, which is believed to be caused by various layers of cellular control. To this end, a combination of both the topdown and bottom-up approaches seems promising to elucidate the effect of genetic interventions. As GEMs allow for incorporating gene, transcript, protein and reaction relationships, they are capable to be efficiently used as scaffolds for omics data integration to refine their reconstruction as well as defining accurate constrains on flux predictions. This will consequently result in not only improved predictions of cellular phenotype or product formations but also will help gaining insight into regulation of metabolism in response to environmental or genetic perturbations. In this regard, advancements in metabolomics data generation and analysis in yeasts (Sailwal et al., 2020) could be employed for Y. lipolytica research and assist in learning about its pathway to further aid in metabolic engineering. Accordingly, while inferring exchange fluxes from extracellular measurements can be used for more accurate FBA simulations, direct integration of intracellular metabolite concentrations withing the FBA framework is challenging due to the quasi-steady state assumption in GEMs (Pandey et al., 2019). 
However, these data can still be used to improve the GEM by finding reactions directionality as well as finding missing functions. Additionally, analysis of metabolomics data alone or in combination with proteomics data, can promise accurate assessment of pathway flux, cofactor imbalance as well as carbon balance diversion which will further aid in finding pathway bottlenecks in strain design (Amer \& Baidoo, 2021).

Integration of transcriptomics data gives insight on whether flux distributions are transcriptionally regulated which in turn represent suitable targets for metabolic engineering to rationally altering fluxes. Although being a more frequently used strategy in omics data integration studies, availability of transcriptomics from different culture conditions and mutant strains will improve the strain design for Y. lipolytica. At the same time, since the regulation of a biological function may occur across the different levels, combination of additional levels of information such as proteomics, metabolomics and fluxomics can be used for better identification of active regulatory paths. Although yet challenging, using GEMs as a scaffold for integration of multiple levels is a foreseeable strategy to be implemented particularly due to advancements of computational algorithms being developed for data integration. However, this will require careful experimental considerations in terms of generating data sets which must be all from the same media, culture condition and strain.

\section{2 | Complete the genome annotation}

A notable obstacle limiting the impact of systems-level metabolic engineering is that many $Y$. lipolytica gene products (ca. $40 \%$ in case of strain W29) are annotated as hypothetical proteins, which can hinder the interpretation of data-driven information as well as predictive efficacy of genome-based models. This need for a more complete annotation of the $Y$. lipolytica genome can at least partially be addressed using available computational approaches, where for example, recent advances in machine learning can accurately predict both translation start sites and functional annotations (G. B. Kim et al., 2020). Artificial neural networks, clustering and other machinelearning algorithms have shown promising results in biosynthetic pathway prediction for a target chemical (Segler et al., 2018) (useful in nonnative chemical productions), prediction of a probability that an enzyme catalyzes a reaction (Mellor et al., 2016) (useful in GEM reconstruction), and different gene expression control parameter prediction such as promoter strength (Jervis et al., 2019) (useful in metabolic engineering). While computational functional genomics inevitably yields a certain proportion of incorrect predictions, in contrast to detailed experimental validation of gene function through molecular biology studies, having a hypothetical functional annotation is arguable more informative than having no annotation for ca. $40 \%$ of the genome. Besides, this highlights the continuing value of low-throughput detailed characterization of gene functions, which should not be all superseded by high-throughput multi-omics approaches.

\subsection{Advances in computational approaches}

Regardless of the incomplete genome annotation, the current knowledge can be used to improve the existing computational models. A robust and accurate metabolic model facilitates successful application but relies on the availability of sufficient experimental data. While the Y. lipolytica GEM has been improved (Xu et al., 2020), this should continue akin to the continuous curation and incorporation of new experimental data in the S. cerevisiae GEM. Specifically, the $S$. cerevisiae GEM has been advanced by integrating enzymatic constraints as well as protein $3 \mathrm{D}$ structures, now rendering this model capable of accurately predicting how single nucleotide variations translate to phenotypic traits (Lu et al., 2019). Such strategies are expected to also be of value for nonconventional yeasts such as $Y$. lipolytica.

The lack of kinetic parameters has precluded wider use of kinetic models of $Y$. lipolytica, but this impediment could be countered through ensemble modeling (EM). Instead of settling on specific parameter values, in EM samples of kinetic parameters are repeatedly drawn from an allowable distribution to generate an ensemble of kinetic models that best describe the system under study (Tran et al., 2008). EM has been used in systems metabolic engineering to among others identify targets for improved ethanol overproduction in Synechocystis sp. PCC 6803 (Nishiguchi et al., 2019; Nishiguchi et al., 2020); overproduction of L-lysine in Escherichia coli (Contador et al., 2009); as well as improved ethanol production in Clostridium autoethanogenum (Greene et al., 2019). Applying this approach in Y. lipolytica might be a valuable strategy to further the understanding of its metabolic dynamics and identify novel strategies for improved production, although it should be noted that selection of ensemble models depends on the availability of (dynamic) experimental data from diverse conditions, which is currently relatively limited for $Y$. lipolytica. In particular dynamic metabolomics measurements would contribute valuable criteria to narrow down the solution space described by ensemble models.

Alternatively, existing GEMs could be expanded to allow for integration of further omics data, thereby yielding more accurate predictions of gene modification targets. A classical GEM can, for example, be expanded by introducing enzymatic constraints, where the usage and efficiency of enzymes is explicitly considered (Sánchez et al., 2017). Such enzyme constrained models (ec-models) do not only yield flux distributions that physiologically more relevant, but they also enable the integration of proteomics data to result in condition-specific models. However, ec-models require enzyme efficiencies as $k_{\text {cat }}$ values that are sparsely available for $Y$. lipolytica. While $k_{\text {cat }}$ values could be estimated from related organisms, it is anticipated that machine-learning will be able to give more accurate predictions (Heckmann et al., 2018). GEMs could also be further expanded towards genome-scale models of metabolism and expression (ME-models), enabling the integration of gene expression and protein synthesis data in GEMs. This approach has been used in, for example, E. coli (O'brien et al., 2013) and S. cerevisiae (Oftadeh et al., 2021), but has not been widely applied to non-model 
microorganisms primarily due to the lack of species-specific detailed knowledge of, for example, protein synthesis.

In sum, the increasing availability of systems-level tools and techniques such as genome modification tools, metabolic models and state-of-the-art "omics" studies, are able to enhance our understanding of unique $Y$. lipolytica features and provide guidance for its improvement. However, it remains imperative that a deeper knowledge of regulation and metabolism in $Y$. lipolytica will help to advance it towards a versatile industrial cell-factory. Largely, this can be accomplished by orthogonal application of systems-level and synthetic biology tools that have been very successful to advance model organisms such as S. cerevisiae into promising cell factories, while its unique characteristics can give Y. lipolytica an advantage. Moreover, as Y. lipolytica can be regarded as a promising host for the biosynthesis of various products, it is expected that elucidation of systems-level behaviors for one product will also positively impact on this yeast as a future host for other products.

\section{ACKNOWLEDGMENT}

We acknowledge funding from the Novo Nordisk Foundation (grant no. NNF10CC1016517) and the Research Council for Environment, Agricultural Sciences and Spatial Planning (Formas, grant no. 201800597).

\section{CONFLICT OF INTERESTS}

The authors declare that there are no conflict of interests.

\section{AUTHOR CONTRIBUTIONS}

Naghmeh Poorinmohammad conceived, wrote, and edited the manuscript. Eduard J. Kerkhoven critically revised and edited the manuscript.

\section{DATA AVAILABILITY STATEMENT}

Data sharing not applicable to this article as no data sets were generated or analyzed during the current study

\section{ORCID}

Eduard J. Kerkhoven (D) https://orcid.org/0000-0002-3593-5792

\section{REFERENCES}

Abdel-Mawgoud, A. M., Markham, K. A., Palmer, C. M., Liu, N. Stephanopoulos, G., \& Alper, H. S. (2018). Metabolic engineering in the host Yarrowia lipolytica. Metabolic Engineering, 50, 192-208. https://doi.org/10.1016/j.ymben.2018.07.016

Agren, R., Otero, J. M., \& Nielsen, J. (2013). Genome-scale modeling enables metabolic engineering of Saccharomyces cerevisiae for succinic acid production. Journal of Industrial Microbiology and Biotechnology, 40(7), 735-747. https://doi.org/10.1007/s10295013-1269-3

Ahn, J. H., Jang, Y. S., \& Lee, S. Y. (2016). Production of succinic acid by metabolically engineered microorganisms. Current Opinion in Biotechnology, 42, 54-66. https://doi.org/10.1016/j.copbio.2016. 02.034
Amer, B., \& Baidoo, E. E. K. (2021). Omics-driven biotechnology for industrial applications. Frontiers in Bioengineering and Biotechnology, 9, 30.

Beckerich, J. M., Boisramé-Baudevin, A., \& Gaillardin, C. (1998). Yarrowia lipolytica: A model organism for protein secretion studies. International Microbiology, 1(2), 123-130. https://doi.org/10.2436/ im.v1i2.57

Beopoulos, A., Chardot, T., \& Nicaud, J. M. (2009). Yarrowia lipolytica: A model and a tool to understand the mechanisms implicated in lipid accumulation. Biochimie, 91(6), 692-696. https://doi.org/10.1016/j. biochi.2009.02.004

Bilal, M., Xu, S., Iqbal, H. M. N., \& Cheng, H. (2020). Yarrowia lipolytica as an emerging biotechnological chassis for functional sugars biosynthesis. Critical Reviews in Food Science and Nutrition, 1-18. https://doi.org/10.1080/10408398.2020.1739000

Blazeck, J., Hill, A., Jamoussi, M., Pan, A., Miller, J., \& Alper, H. S. (2015). Metabolic engineering of Yarrowia lipolytica for itaconic acid production. Metabolic Engineering, 32, 66-73. https://doi.org/10. 1016/j.ymben.2015.09.005

Blazeck, J., Hill, A., Liu, L., Knight, R., Miller, J., Pan, A., Otoupal, P., \& Alper, H. S. (2014). Harnessing Yarrowia lipolytica lipogenesis to create a platform for lipid and biofuel production. Nature Communications, 5(1), 1-10. https://doi.org/10.1038/ncomms4131

Cai, P., Gao, J., \& Zhou, Y. (2019). CRISPR-mediated genome editing in non-conventional yeasts for biotechnological applications. Microbial Cell Factories, 18(1), 63.

Campbell, K., Xia, J., \& Nielsen, J. (2017). The impact of systems biology on bioprocessing. Trends in Biotechnology, 35(12), 1156-1168. https://doi.org/10.1016/j.tibtech.2017.08.011

Carsanba, E., Papanikolaou, S., Fickers, P., \& Erten, H. (2019). Screening various Yarrowia lipolytica strains for citric acid production. Yeast, 36(5), 319-327. https://doi.org/10.1002/yea.3389

Contador, C. A., Rizk, M. L., Asenjo, J. A., \& Liao, J. C. (2009). Ensemble modeling for strain development of L-lysine-producing Escherichia coli. Metabolic Engineering, 11(4-5), 221-233.

Cui, Z., Gao, C., Li, J., Hou, J., Lin, C. S. K., \& Qi, Q. (2017). Engineering of unconventional yeast Yarrowia lipolytica for efficient succinic acid production from glycerol at low $\mathrm{pH}$. Metabolic Engineering, 42, 126-133. https://doi.org/10.1016/j.ymben.2017.06.007

Cui, Z., Zheng, H., Zhang, J., Jiang, Z., Zhu, Z., Liu, X., Qi, Q., \& Hou, J. (2021). Development of a CRISPR/Cas9-mediated homologyindependent tool for targeted genome integration in Yarrowia lipolytica. Applied and Environmental Microbiology, 87(6):e02666-20.

Dahlin, J., Holkenbrink, C., Marella, E. R., Wang, G., Liebal, U., Lieven, C., Weber, D., McCloskey, D., Ebert, B. E., Herrgård, M. J., Blank, L. M., \& Borodina, I. (2019). Multi-omics analysis of fatty alcohol production in engineered yeasts Saccharomyces cerevisiae and Yarrowia lipolytica. Frontiers in Genetics, 10, 747. https://doi.org/10. 3389/fgene.2019.00747

Das, M., Patra, P., \& Ghosh, A. (2020). Metabolic engineering for enhancing microbial biosynthesis of advanced biofuels. Renewable and Sustainable Energy Reviews, 119, 109562. https://doi.org/10. 1016/j.rser.2019.109562

Domínguez, A., Fermiñán, E., \& Gaillardin, C. (2000). Yarrowia lipolytica: An organism amenable to genetic manipulation as a model for analyzing dimorphism in fungi. Contributions to Microbiology, 5, 151-172. https://doi.org/10.1159/000060349

El-Rotail, A. A. M. M., Zhang, L., Li, Y., Liu, S. P., \& Shi, G. Y. (2017). A novel constructed SPT15 mutagenesis library of Saccharomyces cerevisiae by using gTME technique for enhanced ethanol production. AMB Express, 7(1), 111. https://doi.org/10.1186/s13568-017-0400-7

Fakas, S. (2017). Lipid biosynthesis in yeasts: A comparison of the lipid biosynthetic pathway between the model nonoleaginous yeast Saccharomyces cerevisiae and the model oleaginous yeast Yarrowia 
lipolytica. Engineering in Life Sciences, 17(3), 292-302. https://doi.org/ 10.1002/elsc.201600040

Fickers, P., Benetti, P. H., Waché, Y., Marty, A., Mauersberger, S., Smit, M. S., \& Nicaud, J. M. (2005). Hydrophobic substrate utilisation by the yeast Yarrowia lipolytica, and its potential applications. FEMS Yeast Research, 5(6-7), 527-543. https://doi.org/10.1016/j.femsyr. 2004.09.004

Finogenova, T. V., Morgunov, I. G., Kamzolova, S. V., \& Chernyavskaya, O. G. (2005). Organic acid production by the yeast Yarrowia lipolytica: A review of prospects. Applied Biochemistry and Microbiology, 41(5), 418-425. https://doi.org/10.1007/s10438-0050076-7

Foster, C. J., Wang, L., Dinh, H. V., Suthers, P. F., \& Maranas, C. D. (2021). Building kinetic models for metabolic engineering. Current Opinion in Biotechnology, 67, 35-41. https://doi.org/10.1016/j.copbio.2020. 11.010

Ganesan, V., Spagnuolo, M., Agrawal, A., Smith, S., Gao, D., \& Blenner, M. (2019). Advances and opportunities in gene editing and gene regulation technology for Yarrowia lipolytica. Microbial Cell Factories, 18(1), 1-9. https://doi.org/10.1186/s12934-019-1259-x

Gao, S., Tong, Y., Wen, Z., Zhu, L., Ge, M., Chen, D., Jiang, Y., \& Yang, S. (2016). Multiplex gene editing of the Yarrowia lipolytica genome using the CRISPR-Cas9 system. Journal of Industrial Microbiology and Biotechnology, 43(8), 1085-1093. https://doi.org/10.1007/s10295016-1789-8

Greene, J., Daniell, J., Köpke, M., Broadbelt, L., \& Tyo, K. E. J. (2019). Kinetic ensemble model of gas fermenting Clostridium autoethanogenum for improved ethanol production. Biochemical Engineering Journal, 148, 46-56.

Groenewald, M., Boekhout, T., Neuvéglise, C., Gaillardin, C., Van Dijck, P. W. M., \& Wyss, M. (2014). Yarrowia lipolytica: Safety assessment of an oleaginous yeast with a great industrial potential. Critical Reviews in Microbiology, 40(3), 187-206. https://doi.org/10. 3109/1040841X.2013.770386

Gu, C., Kim, G. B., Kim, W. J., Kim, H. U., \& Lee, S. Y. (2019). Current status and applications of genome-scale metabolic models. Genome Biology, 20(1), 121. https://doi.org/10.1186/s13059-019-1730-3

Guo, H., Liu, P., Madzak, C., Du, G., Zhou, J., \& Chen, J. (2015). Identification and application of keto acids transporters in Yarrowia lipolytica. Scientific Reports, 5(1), 8138. https://doi.org/10. 1038/srep08138

Guo, H., Su, S., Madzak, C., Zhou, J., Chen, H., \& Chen, G. (2016). Applying pathway engineering to enhance production of alphaketoglutarate in Yarrowia lipolytica. Applied Microbiology and Biotechnology, 100(23), 9875-9884. https://doi.org/10.1007/ s00253-016-7913-x

Guo, Z., Borsenberger, V., Croux, C., Duquesne, S., Truan, G., Marty, A., \& Bordes, F. (2020). An artificial chromosome yIAC enables efficient assembly of multiple genes in Yarrowia lipolytica for biomanufacturing. Communications Biology, 3(1), 199. https://doi. org/10.1038/s42003-020-0936-y

Harder, B. J., Bettenbrock, K., \& Klamt, S. (2016). Model-based metabolic engineering enables high yield itaconic acid production by Escherichia coli. Metabolic Engineering, 38, 29-37. https://doi.org/10. 1016/j.ymben.2016.05.008

Heckmann, D., Lloyd, C. J., Mih, N., Ha, Y., Zielinski, D. C., Haiman, Z. B., Desouki, A. A., Lercher, M. J., \& Palsson, B. O. (2018). Machine learning applied to enzyme turnover numbers reveals protein structural correlates and improves metabolic models. Nature Communications, 9(1), 1-10.

Holkenbrink, C., Dam, M. I., Kildegaard, K. R., Beder, J., Dahlin, J., Doménech Belda, D., \& Borodina, I. (2018). EasyCloneYALI: CRISPR/ Cas9-based synthetic toolbox for engineering of the yeast Yarrowia lipolytica. Biotechnology Journal, 13(9), 1700543. https://doi.org/10. 1002/biot.201700543
Hubbard, A. H., Zhang, X., Jastrebski, S., Lamont, S. J., Singh, A., \& Schmidt, C. J. (2018). Identifying mechanisms of regulation to model carbon flux during heat stress and generate testable hypotheses. PLoS One, 13(10), e0205824.

Jervis, A. J., Carbonell, P., Taylor, S., Sung, R., Dunstan, M. S., Robinson, C. J., Breitling, R., Takano, E., \& Scrutton, N. S. (2019). SelProm: A queryable and predictive expression vector selection tool for Escherichia coli. ACS Synthetic Biology, 8(7), 1478-1483. https://doi.org/10.1021/acssynbio.8b00399

Kamzolova, S. V., \& Morgunov, I. G. (2017). Metabolic peculiarities of the citric acid overproduction from glucose in yeasts Yarrowia lipolytica. Bioresource Technology, 243, 433-440. https://doi.org/10.1016/j. biortech.2017.06.146

Kanamasa, S., Dwiarti, L., Okabe, M., \& Park, E. Y. (2008). Cloning and functional characterization of the cis-aconitic acid decarboxylase (CAD) gene from Aspergillus terreus. Applied Microbiology and Biotechnology, 80(2), 223-229. https://doi.org/10.1007/s00253008-1523-1

Kavšček, M., Bhutada, G., Madl, T., \& Natter, K. (2015). Optimization of lipid production with a genome-scale model of Yarrowia lipolytica. BMC Systems Biology, 9(1), 72. https://doi.org/10.1186/s12918-0150217-4

Kerkhoven, E. J., Kim, Y. M., Wei, S., Nicora, C. D., Fillmore, T. L., Purvine, S. O., Webb-Robertson, B. J., Smith, R. D., Baker, S. E., Metz, T. O., \& Nielsena, J. (2017). Leucine biosynthesis is involved in regulating high lipid accumulation in Yarrowia lipolytica. mBio, 8(3), e00857-17. https://doi.org/10.1128/mBio.00857-17

Kerkhoven, E. J., Pomraning, K. R., Baker, S. E., \& Nielsen, J. (2016). Regulation of amino-acid metabolism controls flux to lipid accumulation in Yarrowia lipolytica. NPJ Systems Biology and Applications, 2, 16005. https://doi.org/10.1038/npjsba.2016.5

Kim, G. B., Kim, W. J., Kim, H. U., \& Lee, S. Y. (2020). Machine learning applications in systems metabolic engineering. Current Opinion in Biotechnology, 64, 1-9. https://doi.org/10.1016/j.copbio.2019. 08.010

Kim, M., Park, B. G., Kim, E.-J., Kim, J., \& Kim, B.-G. (2019). In silico identification of metabolic engineering strategies for improved lipid production in Yarrowia lipolytica by genome-scale metabolic modeling. Biotechnology for Biofuels, 12(1), 187. https://doi.org/10. 1186/s13068-019-1518-4

Lazar, Z., Liu, N., \& Stephanopoulos, G. (2018). Holistic approaches in lipid production by Yarrowia lipolytica. Trends in Biotechnology, 36(11), 1157-1170. https://doi.org/10.1016/j.tibtech.2018.06.007

Ledesma-Amaro, R., \& Nicaud, J. M. (2016). Yarrowia lipolytica as a biotechnological chassis to produce usual and unusual fatty acids. Progress in Lipid Research, 61, 40-50. https://doi.org/10.1016/j. plipres.2015.12.001

Leplat, C., Nicaud, J. M., \& Rossignol, T. (2018). Overexpression screen reveals transcription factors involved in lipid accumulation in Yarrowia lipolytica. FEMS Yeast Research, 18(5), foy037 https://doi. org/10.1093/femsyr/foy037

Li, D., Wu, Y., Wei, P., Gao, X., Li, M., Zhang, C., Zhou, Z., \& Lu, W. (2020). Metabolic engineering of Yarrowia lipolytica for heterologous oleanolic acid production. Chemical Engineering Science, 218, 115529. https://doi.org/10.1016/j.ces.2020.115529

Liu, H., Wang, F., Deng, L., \& Xu, P. (2020). Genetic and bioprocess engineering to improve squalene production in Yarrowia lipolytica. Bioresource Technology, 317, 123991.

Liu, L., Markham, K., Blazeck, J., Zhou, N., Leon, D., Otoupal, P., \& Alper, H. S. (2015). Surveying the lipogenesis landscape in Yarrowia lipolytica through understanding the function of a Mga2p regulatory protein mutant. Metabolic Engineering, 31, 102-111.

Liu, L., Pan, A., Spofford, C., Zhou, N., \& Alper, H. S. (2015). An evolutionary metabolic engineering approach for enhancing lipogenesis in Yarrowia lipolytica. Metabolic Engineering, 29, 36-45. 
Liu, N., Qiao, K., \& Stephanopoulos, G. (2016). 13C Metabolic flux analysis of acetate conversion to lipids by Yarrowia lipolytica. Metabolic Engineering, 38, 86-97. https://doi.org/10.1016/j.ymben.2016.06.006

Liu, N., Santala, S., \& Stephanopoulos, G. (2020). Mixed carbon substrates: A necessary nuisance or a missed opportunity? Current Opinion in Biotechnology, 62, 15-21.

Loira, N., Dulermo, T., Nicaud, J. M., \& Sherman, D. J. (2012). A genomescale metabolic model of the lipid-accumulating yeast Yarrowia lipolytica. BMC Systems Biology, 6(1), 35. https://doi.org/10.1186/ 1752-0509-6-35

Lu, H., Li, F., Sánchez, B. J., Zhu, Z., Li, G., Domenzain, I., Marcišauskas, S., Anton, P. M., Lappa, D., \& Lieven, C. (2019). A consensus S. cerevisiae metabolic model Yeast8 and its ecosystem for comprehensively probing cellular metabolism. Nature Communications, 10(1), 1-13.

Madzak, C. (2018). Engineering Yarrowia lipolytica for use in biotechnological applications: A review of major achievements and recent innovations. Molecular Biotechnology, 60(8), 621-635. https:// doi.org/10.1007/s12033-018-0093-4

Markham, K. A., \& Alper, H. S. (2018). Synthetic biology expands the industrial potential of Yarrowia lipolytica. Trends in Biotechnology, 36(10), 1085-1095. https://doi.org/10.1016/j.tibtech.2018.05.004

Mellor, J., Grigoras, I., Carbonell, P., \& Faulon, J. L. (2016). Semisupervised Gaussian process for automated enzyme search. ACS Synthetic Biology, 5(6), 518-528. https://doi.org/10.1021/acssynbio.5b00294

Miller, K. K., \& Alper, H. S. (2019). Yarrowia lipolytica: More than an oleaginous workhorse. Applied Microbiology and Biotechnology, 103(23-24), 9251-9262. https://doi.org/10.1007/s00253-01910200-x

Mishra, P., Lee, N. R., Lakshmanan, M., Kim, M., Kim, B. G., \& Lee, D. Y. (2018). Genome-scale model-driven strain design for dicarboxylic acid production in Yarrowia lipolytica. BMC Systems Biology, 12(2), 12. https://doi.org/10.1186/s12918-018-0542-5

Morin, N., Cescut, J., Beopoulos, A., Lelandais, G., Le Berre, V., Uribelarrea, J. L., Molina-Jouve, C., \& Nicaud, J. M. (2011). Transcriptomic analyses during the transition from biomass production to lipid accumulation in the oleaginous yeast Yarrowia lipolytica. PLoS One, 6(11), 27966. https://doi.org/10.1371/journal. pone.0027966

Nishiguchi, H., Hiasa, N., Uebayashi, K., Liao, J., Shimizu, H., \& Matsuda, F. (2019). Transomics data-driven, ensemble kinetic modeling for system-level understanding and engineering of the cyanobacteria central metabolism. Metabolic Engineering, 52, 273-283.

Nishiguchi, H., Liao, J., Shimizu, H., \& Matsuda, F. (2020). Novel allosteric inhibition of phosphoribulokinase identified by ensemble kinetic modeling of Synechocystis sp. PCC 6803 metabolism. Metabolic Engineering Communications, 11, e00153.

O'brien, E. J., Lerman, J. A., Chang, R. L., Hyduke, D. R., \& Palsson, B. Ø. (2013). Genome-scale models of metabolism and gene expression extend and refine growth phenotype prediction. Molecular Systems Biology, 9(1), 693.

Oftadeh, O., Salvy, P., Masid, M., Curvat, M., Miskovic, L., \& Hatzimanikatis, V. (2021). A genome-scale metabolic model of Saccharomyces cerevisiae that integrates expression constraints and reaction thermodynamics. BioRxiv.

Otero, J. M., Cimini, D., Patil, K. R., Poulsen, S. G., Olsson, L., \& Nielsen, J. (2013). Industrial systems biology of Saccharomyces cerevisiae enables novel succinic acid cell factory. PLoS One, 8(1), 54144. https://doi.org/10.1371/journal.pone.0054144

Pan, P., \& Hua, Q. (2012). Reconstruction and in silico analysis of metabolic network for an oleaginous yeast, Yarrowia lipolytica. PLoS One, 7(12), e51535. https://doi.org/10.1371/journal.pone.0051535

Pandey, V., Gardiol, D. H., Chiappino-Pepe, A., \& Hatzimanikatis, V. (2019). TEX-FBA: A constraint-based method for integrating gene expression, thermodynamics, and metabolomics data into genomescale metabolic models. BioRxiv, 536235.
Patra, P., Das, M., Kundu, P., \& Ghosh, A. (2021). Recent advances in systems and synthetic biology approaches for developing novel cellfactories in non-conventional yeasts. Biotechnology Advances, 47, 107695.

Pomraning, K. R., Kim, Y. M., Nicora, C. D., Chu, R. K., Bredeweg, E. L., Purvine, S. O., Hu, D., Metz, T. O., \& Baker, S. E. (2016). Multi-omics analysis reveals regulators of the response to nitrogen limitation in Yarrowia lipolytica. BMC Genomics, 17(1), 138. https://doi.org/10. 1186/s12864-016-2471-2

Pomraning, K. R., Wei, S., Karagiosis, S. A., Kim, Y. M., Dohnalkova, A. C., Arey, B. W., Bredeweg, E. L., Orr, G., Metz, T. O., \& Baker, S. E. (2015). Comprehensive metabolomic, lipidomic and microscopic profiling of Yarrowia lipolytica during lipid accumulation identifies targets for increased lipogenesis. PLoS One, 10(4), 0123188. https:// doi.org/10.1371/journal.pone.0123188

Ramesh, A., Ong, T., Garcia, J. A., Adams, J., \& Wheeldon, I. (2020). Guide RNA engineering enables dual purpose CRISPR-Cpf1 for simultaneous gene editing and gene regulation in Yarrowia lipolytica. ACS Synthetic Biology, 9(4), 967-971. https://doi.org/10. 1021/acssynbio.9b00498

Raschmanová, H., Weninger, A., Glieder, A., Kovar, K., \& Vogl, T. (2018). Implementing CRISPR-Cas technologies in conventional and nonconventional yeasts: Current state and future prospects. Biotechnology Advances, 36(3), 641-665.

Ritala, A., Häkkinen, S. T., Toivari, M., \& Wiebe, M. G. (2017). Single cell protein-state-of-the-art, industrial landscape and patents 20012016. Frontiers in Microbiology, 8(OCT), 2009. https://doi.org/10. 3389/fmicb.2017.02009

Robert, T., \& Friebel, S. (2016). Itaconic acid-a versatile building block for renewable polyesters with enhanced functionality. Green Chemistry, 18(10), 2922-2934. https://doi.org/10.1039/c6gc00605a

Robles-Rodriguez, C. E., Bideaux, C., Guillouet, S. E., Gorret, N., Cescut, J., Uribelarrea, J. L., Molina-Jouve, C., Roux, G., \& Aceves-Lara, C. A. (2017). Dynamic metabolic modeling of lipid accumulation and citric acid production by Yarrowia lipolytica. Computers and Chemical Engineering, 100, 139-152. https://doi.org/10.1016/j.compchemeng.2017.02.013

Sabra, W., Bommareddy, R. R., Maheshwari, G., Papanikolaou, S., \& Zeng, A. P. (2017). Substrates and oxygen dependent citric acid production by Yarrowia lipolytica: Insights through transcriptome and fluxome analyses. Microbial Cell Factories, 16(1), 78. https://doi. org/10.1186/s12934-017-0690-0

Sailwal, M., Das, A. J., Gazara, R. K., Dasgupta, D., Bhaskar, T., Hazra, S., \& Ghosh, D. (2020). Connecting the dots: Advances in modern metabolomics and its application in yeast system. Biotechnology Advances, 44, 107616.

Sánchez, B. J., Zhang, C., Nilsson, A., Lahtvee, P., Kerkhoven, E. J., \& Nielsen, J. (2017). Improving the phenotype predictions of a yeast genome-scale metabolic model by incorporating enzymatic constraints. Molecular Systems Biology, 13(8), 935.

Schwartz, C., Curtis, N., Löbs, A. K., \& Wheeldon, I. (2018). Multiplexed CRISPR activation of cryptic sugar metabolism enables Yarrowia lipolytica growth on cellobiose. Biotechnology Journal, 13(9), 1700584. https://doi.org/10.1002/biot.201700584

Schwartz, C., Frogue, K., Ramesh, A., Misa, J., \& Wheeldon, I. (2017). CRISPRi repression of nonhomologous end-joining for enhanced genome engineering via homologous recombination in Yarrowia lipolytica. Biotechnology and Bioengineering, 114(12), 2896-2906. https://doi.org/10.1002/bit.26404

Schwartz, C. M., Hussain, M. S., Blenner, M., \& Wheeldon, I. (2016). Synthetic RNA polymerase III promoters facilitate high-efficiency CRISPR-Cas9mediated genome editing in Yarrowia lipolytica. ACS Synthetic Biology, 5(4), 356-359. https://doi.org/10.1021/acssynbio.5b00162

Segler, M. H. S., Preuss, M., \& Waller, M. P. (2018). Planning chemical syntheses with deep neural networks and symbolic Al. Nature, 555(7698), 604-610. https://doi.org/10.1038/nature25978 
Seip, J., Jackson, R., He, H., Zhu, Q., \& Hong, S.-P. (2013). Snf1 is a regulator of lipid accumulation in Yarrowia lipolytica. Applied and Environmental Microbiology, 79(23), 7360-7370.

Shi, S., Chen, Y., Siewers, V., \& Nielsen, J. (2014). Improving production of malonyl coenzyme A-derived metabolites by abolishing Snf1dependent regulation of Acc1. mBio, 5, 3-14.

Soong, Y. V., Liu, N., Yoon, S., Lawton, C., \& Xie, D. (2019). Cellular and metabolic engineering of oleaginous yeast Yarrowia lipolytica for bioconversion of hydrophobic substrates into high-value products. Engineering in Life Sciences, 19(6), 423-443.

Soudier, P., Larroude, M., Celińska, E., Rossignol, T., \& Nicaud, J.-M. (2019). Selection of heterologous protein-producing strains in Yarrowia lipolytica. Recombinant Protein Production in Yeast, 1923, 153-168.

Tan, F., Wu, B., Dai, L., Qin, H., Shui, Z., Wang, J., Zhu, Q., Hu, G., \& He, M. (2016). Using global transcription machinery engineering (gTME) to improve ethanol tolerance of Zymomonas mobilis. Microbial Cell Factories, 15(1), 4. https://doi.org/10.1186/s12934-015-0398-y

Timmis, K., de Vos, W. M., Ramos, J. L., Vlaeminck, S. E., Prieto, A., Danchin, A., Verstraete, W., de Lorenzo, V., Lee, S. Y., Brüssow, H., Timmis, J. K., \& Singh, B. K. (2017). The contribution of microbial biotechnology to sustainable development goals. Microbial Biotechnology, 10(5), 984-987. https://doi.org/10.1111/1751-7915. 12818

Tran, L. M., Rizk, M. L., \& Liao, J. C. (2008). Ensemble modeling of metabolic networks. Biophysical Journal, 95(12), 5606-5617.

Trébulle, P., Nicaud, J. M., Leplat, C., \& Elati, M. (2017). Inference and interrogation of a coregulatory network in the context of lipid accumulation in Yarrowia lipolytica. NPJ Systems Biology and Applications, 3(1), 1-8. https://doi.org/10.1038/s41540-017-0024-1

Walker, C., Ryu, S., Giannone, R. J., Garcia, S., \& Trinh, C. T. (2020). Understanding and eliminating the detrimental effect of thiamine deficiency on the oleaginous yeast Yarrowia lipolytica. Applied and Environmental Microbiology, 86(3), https://doi.org/10.1128/AEM. 02299-19

Wang, G., Li, D., Miao, Z., Zhang, S., Liang, W., \& Liu, L. (2018). Comparative transcriptome analysis reveals multiple functions for Mhy1p in lipid biosynthesis in the oleaginous yeast Yarrowia lipolytica. Biochimica et Biophysica Acta - Molecular and Cell Biology of Lipids, 1863(1), 81-90. https://doi.org/10.1016/j.bbalip.2017. 10.003

Wang, M., Liu, G.-N., Liu, H., Zhang, L., Li, B.-Z., Li, X., Liu, D., \& Yuan, Y.-J. (2018). Engineering global transcription to tune lipophilic properties in Yarrowia lipolytica. Biotechnology for Biofuels, 11(1), 115. https:// doi.org/10.1186/s13068-018-1114-z

Wang, Z.-P., Xu, H.-M., Wang, G.-Y., Chi, Z., \& Chi, Z.-M. (2013). Disruption of the MIG1 gene enhances lipid biosynthesis in the oleaginous yeast Yarrowia lipolytica ACA-DC 50109. Biochimica et Biophysica Acta (BBA)-Molecular and Cell Biology of Lipids, 1831(4), 675-682.

Wasylenko, T. M., Ahn, W. S., \& Stephanopoulos, G. (2015). The oxidative pentose phosphate pathway is the primary source of NADPH for lipid overproduction from glucose in Yarrowia lipolytica. Metabolic Engineering, 30, 27-39. https://doi.org/10.1016/j.ymben.2015. 02.007

Wei, S., Jian, X., Chen, J., Zhang, C., \& Hua, Q. (2017). Reconstruction of genome-scale metabolic model of Yarrowia lipolytica and its application in overproduction of triacylglycerol. Bioresources and Bioprocessing, 4(1), 51. https://doi.org/10.1186/s40643-017-0180-6

Xie, D., Jackson, E. N., \& Zhu, Q. (2015). Sustainable source of omega-3 eicosapentaenoic acid from metabolically engineered Yarrowia lipolytica: From fundamental research to commercial production. Applied Microbiology and Biotechnology, 99(4), 1599-1610. https:// doi.org/10.1007/s00253-014-6318-y
Xu, Y., Holic, R., \& Hua, Q. (2020). Comparison and analysis of published genome-scale metabolic models of Yarrowia lipolytica. Biotechnology and Bioprocess Engineering, 25(1), 53-61.

Yang, L. B., Dai, X. M., Zheng, Z. Y., Zhu, L., Zhan, X. B., \& Lin, C. C. (2015). Proteomic analysis of erythritol-producing yarrowia lipolytica from glycerol in response to osmotic pressure. Journal of Microbiology and Biotechnology, 25(7), 1056-1069. https://doi.org/10.4014/jmb.1412. 12026

Yang, L. B., Zhan, X. B., Zheng, Z. Y., Wu, J. R., Gao, M. J., \& Lin, C. C. (2014). A novel osmotic pressure control fed-batch fermentation strategy for improvement of erythritol production by Yarrowia lipolytica from glycerol. Bioresource Technology, 151, 120-127. https://doi.org/10.1016/j.biortech.2013.10.031

Yang, Z., Edwards, H., \& Xu, P. (2020). CRISPR-Cas12a/Cpf1-assisted precise, efficient and multiplexed genome-editing in Yarrowia lipolytica. Metabolic Engineering Communications, 10, e00112. https://doi.org/10.1016/j.mec.2019.e00112

Yuzbashev, T. V., Bondarenko, P. Y., Sobolevskaya, T. I., Yuzbasheva, E. Y., Laptev, I. A., Kachala, V. V., Fedorov, A. S., Vybornaya, T. V., Larina, A. S., \& Sineoky, S. P. (2016). Metabolic evolution and $13 \mathrm{C}$ flux analysis of a succinate dehydrogenase deficient strain of . Biotechnology and Bioengineering, 113(11), 2425-2432. https://doi. org/10.1002/bit.26007

Yuzbashev, T. V., Yuzbasheva, E. Y., Sobolevskaya, T. I., Laptev, I. A., Vybornaya, T. V., Larina, A. S., Matsui, K., Fukui, K., \& Sineoky, S. P. (2010). Production of succinic acid at low pH by a recombinant strain of the aerobic yeast Yarrowia lipolytica. Biotechnology and Bioengineering, 107(4), 673-682. https://doi.org/10.1002/bit.22859

Zeng, W., Fang, F., Liu, S., Du, G., Chen, J., \& Zhou, J. (2016). Comparative genomics analysis of a series of Yarrowia lipolytica WSH-ZO6 mutants with varied capacity for $\alpha$-ketoglutarate production. Journal of Biotechnology, 239, 76-82. https://doi.org/10.1016/j. jbiotec.2016.10.008

Zhang, J.-L., Bai, Q.-Y., Peng, Y.-Z., Fan, J., Jin, C.-C., Cao, Y.-X., \& Yuan, Y.-J. (2020). High production of triterpenoids in Yarrowia lipolytica through manipulation of lipid components. Biotechnology for Biofuels, 13(1), 1-13.

Zhang, J.-L., Cao, Y.-X., Peng, Y.-Z., Jin, C.-C., Bai, Q.-Y., Zhang, R.-S., Liu, D., \& Yuan, Y.-J. (2019). High production of fatty alcohols in Yarrowia lipolytica by coordination with glycolysis. Science China Chemistry, 62(8), 1007-1016. https://doi.org/10.1007/s11426-0199456-y

Zhao, C., Gu, D., Nambou, K., Wei, L., Chen, J., Imanaka, T., \& Hua, Q. (2015). Metabolome analysis and pathway abundance profiling of Yarrowia lipolytica cultivated on different carbon sources. Journal of Biotechnology, 206, 42-51. https://doi.org/10.1016/j.jbiotec.2015. 04.005

Zhou, Q., Jiao, L., Li, W., Hu, Z., Li, Y., Zhang, H., Xu, L., \& Yan, Y. (2021). A novel and effective Cre/lox-based genetic tool for repeated, targeted and markerless gene integration. BioRxiv, 2020(12.30), 424803. https://doi.org/10.1101/2020.12.30.424803

Zhu, Z., Zhang, S., Liu, H., Shen, H., Lin, X., Yang, F., Zhou, Y. J., Jin, G., Ye, M., \& Zou, H. (2012). A multi-omic map of the lipid-producing yeast Rhodosporidium toruloides. Nature Communications, 3(1), 1-12.

How to cite this article: Poorinmohammad, N., Kerkhoven, E. J. (2021). Systems-level approaches for understanding and engineering of the oleaginous cell factory Yarrowia lipolytica. Biotechnology and Bioengineering, 1-15.

https://doi.org/10.1002/bit.27859 